\title{
Physiological Relevance of a Minimal Model in Healthy Pigs Lung
}

\author{
Yeong Shiong Chiew*, Thomas Desaive** Bernard Lambermont**, Nathalie Janssen**, \\ Geoffrey M Shaw***, Christoph Schranz****, Knut Möller****, J.Geoffrey Chase* \\ *Department of Mechanical Engineering, University of Canterbury, \\ Christchurch, New Zealand (e-mail: Yeong.Chiew@pg.canterbury.ac.nz). \\ **Hemodynamic Research Center, HemoLiege, University of Liege, \\ Liege, Belgium (email: Tdesaive@ulg.ac.be) \\ *** Intensive Care Unit, Christchurch Hospital, \\ Christchurch, New Zealand, (e-mail: Geoff.Shaw@cdhb.govt.nz) \\ **** Institute for Technical Medicine, Furtwangen University, \\ Villingen-Schwenningen, Germany, (e-mail: scc@hs-furtwangen.de)
}

\begin{abstract}
Mechanical Ventilation (MV) is the primary form of support for acute lung injury and acute respiratory distress (ALI/ARDS) patients. However, intra- and inter- patient variability makes consistent care difficult and encourages the use of modeling approach to assist in patient-specific MV treatment. A minimal model is tested in 9 healthy pigs and subsequently in 3 ARDS pigs to evaluate its performance and physiological relevance for both conditions, as well as change in condition. The model estimates the mean of threshold opening pressure (TOP), mean threshold closing pressure (TCP) and standard deviation (SD) of both the TOP and TCP distribution for the animal at each different condition. The median percentage fitting error during inflation healthy and ARDS is less than $7.0 \%$ across all animals, indicating that the model is capable of capturing the fundamental lung mechanics during PEEP increase. An increase of mean TOP is observed between healthy and ARDS animal, suggesting higher pressure is required to recruit injured and collapsed lung during ARDS. The minimal model was able to capture the characteristic of both healthy and ARDS lungs, and it is able to track disease progression between states. It thus offers potentially opportunity to guide clinical decision making.
\end{abstract}

Keywords: ALI, ARDS, Healthy lung, minimal model, mechanical ventilation, decision making.

\section{INTRODUCTION}

Mechanical ventilation (MV) is used in the intensive care unit (ICU) to ventilate patients with respiratory failure. Patients with acute lung injury or acute respiratory distress syndrome (ALI/ARDS) are dependent on MV for life support and treatment. ALI/ ARDS is extremely heterogeneous with significant inter- and intra- patient variation, which means patient-specific treatment is required to optimise the outcome. Computer modelling is increasingly used to identify and characterise patient-specific lung condition and guide clinical decision (Rees et al., 2006, Chase et al., 2011, Schranz et al., 2011).

ALI/ARDS is associated with fluid filled lungs (oedema), alveolar collapse and instability, resulting in reduced lung compliance. A recruitment model that characterised the ARDS lung was described by Hickling (Hickling, 1998). It assumes the lung consists of a collection of lung units (alveoli) that are either healthy or injured. Healthy alveoli are normally open and consist of a residual volume that can be filled up easily with air. ARDS lung consist of injured alveoli that are often collapsed and have relatively no residual volume.

However, collapsed alveoli can be opened (recruited) with added pressure through mechanical ventilation. Once the alveoli are opened, they assume a volume similar to healthy alveoli. The opening and closing of each collapsed alveoli are governed by an effective threshold opening pressure (TOP) and threshold closing pressure (TCP) (Crotti et al., 2001, Pelosi et al., 2001). Thus, estimating these parameters in a patient provides an unique insight to patient-specific physiological condition, response to different MV treatment, and the opportunity to optimise patient-specific MV settings (Sundaresan et al., 2011).

A healthy spontaneously breathing lung normally has no collapsed alveoli. Thus, recruitment models are considered to only be applicable for estimating lung mechanics in ALI/ ARDS or similar cases. A minimal model was proposed by Sundaresan et al capable of monitoring the patient-disease state and to guide MV therapy in the ICU (Sundaresan et al., 2009, Sundaresan et al., 2011). It was able to identify physiological relevant parameters that characterised patientspecific conditions. However, the model is only used and tested in ALI/ARDS patients, and the model is yet to be validated in healthy lung.

In this study, an animal trial is carried out to test the physiological relevance and investigate the performance of the minimal model in both healthy and ARDS lungs. We hypothesise that the minimal model is an extension of a recruitment model and able to represent not just a diseased lung, but healthy lungs, as well. 


\section{METHODS}

\subsection{Animal Preparation}

Experimental piglets were premedicated with tiletamin zolazepam $5 \mathrm{mg} / \mathrm{kg}$ and subsequently anaesthetised by a continuous infusion of sufentanil $0.5 \mu \mathrm{g} / \mathrm{kg} / \mathrm{h}$, pentobarbital $5 \mathrm{mg} / \mathrm{kg} / \mathrm{h}$ and cisatracurium $2 \mathrm{mg} / \mathrm{kg} / \mathrm{h}$. They were ventilated through tracheotomy under volume control (Tidal volume, $\mathrm{Vt}$ $\cong 300 \mathrm{ml}$ ) with a inspired oxygen fraction $(\mathrm{FiO} 2)$ of 0.5 using Engström CareStation ventilator (Datex, General Electric, Finland).

\subsection{Protocol-based Manoeuvre}

Each animal underwent a protocol-based step-wise PEEP (positive end-expiratory pressure) increase recruitment manoeuvre (RM). Animals were initially ventilated at baseline PEEP of $5 \mathrm{cmH}_{2} \mathrm{O}$, and PEEP was increased with a $5 \mathrm{cmH}_{2} \mathrm{O}$ step until $20 \mathrm{cmH}_{2} \mathrm{O}$. Other ventilator settings were maintained throughout the trial. During RM, each PEEP level was maintained for 10 15 breaths until stabilisation before increasing to a higher PEEP level. Fig 1 shows an example of the continuously recorded airway pressure and flow during the RM.
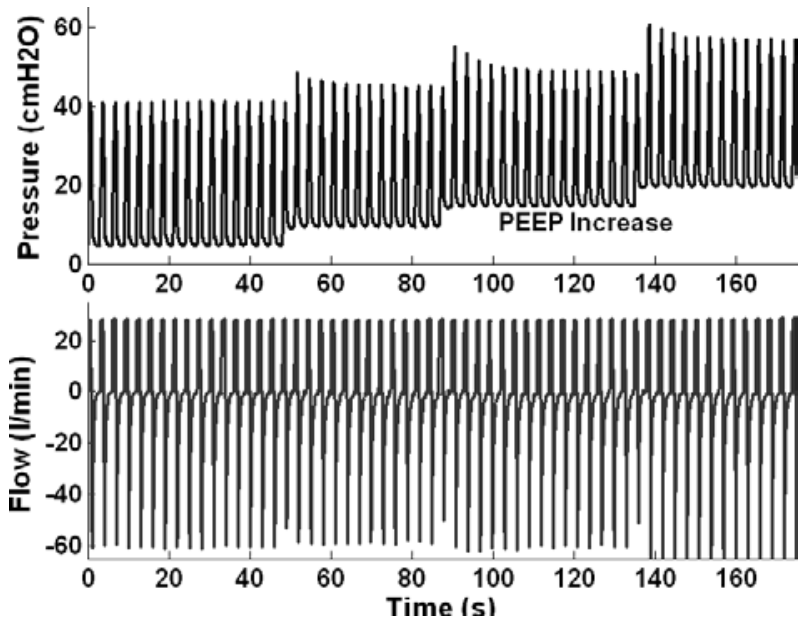

Fig. 1. Continuous pressure and flow profile during recruitment manoeuvre (RM).

After RM, the PEEP was decreased step-wise until baseline PEEP at $5 \mathrm{cmH}_{2} \mathrm{O}$. At this PEEP, the healthy pigs were then injected with oleic acid to induce ARDS. Oleic acid was administrated slowly at $0.1 \mathrm{ml}$ for every 10 minutes interval until $0.1 \mathrm{ml} / \mathrm{kg}$ of the animal's weight. Arterial blood gases were monitored hourly, and, once diagnosed with ARDS, the animal underwent second RM. In this study, ARDS criteria is limited to monitoring arterial blood gas of the animal with PF ratio $<200 \mathrm{mmHg}$. All experimental procedure, protocols and the use of data in this study were reviewed and approved by the Ethics Committee of the University of Liege Medical Faculty.

\subsection{Model Fitting and Data Analysis}

A stabilised representative breath was selected at each PEEP level to plot pressure-volume (PV) curve. This breath is selected from the last 2 breaths at each PEEP level, with the assumption of viscoelastic stabilisation has occurred after PEEP increase. The volume increase with PEEP increase was estimated through post processing of continuous flow and volume profiles. An example of post-processed PV curves is shown in Fig 2. The change in volume due to increased PEEP is evident.

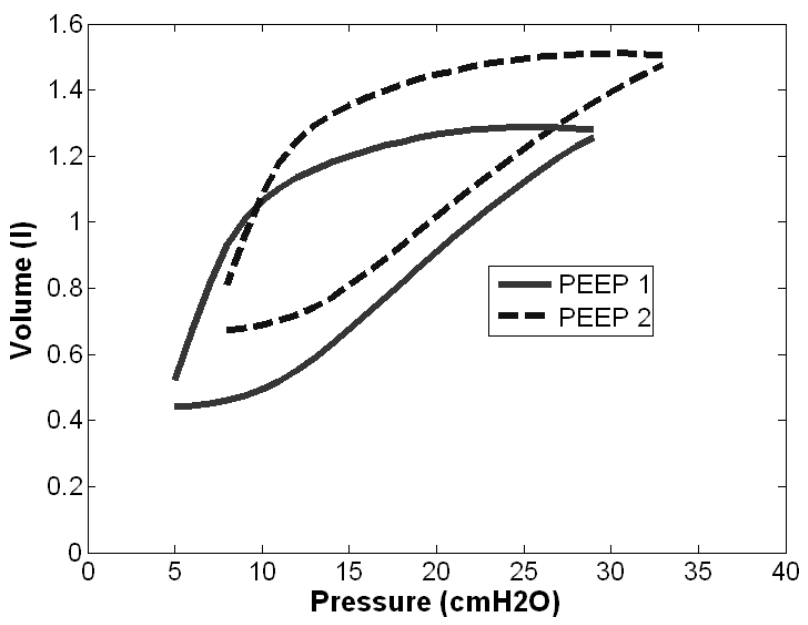

Fig. 2. Example of PV loops with volume change due to PEEP change.

A minimal model (Sundaresan et al., 2009) was used to fit the PV curves and estimate the mean threshold opening pressure (TOP), mean threshold closing pressure (TCP) and standard deviation (SD) of the both TOP and TCP distribution. The minimal model is based on concept of recruitment (Hickling, 1998), with the lung modelled as a collection of lung units $(0.01 \mathrm{ml}$ per lung unit) which are either open or collapsed.

During inflation, if airway pressure is above a lung unit's effective TOP, the lung unit will assume a lung unit volume. Each opened unit volume is added to form the pressure volume (PV) curve during inflation. If the airway pressure during deflation drops below an effective closing pressure, the lung unit collapses. Each lung unit has a different effective opening pressure and closing pressure, and it is assumed that they are normally distributed (Pelosi et al., 2001, Crotti et al., 2001).

In a TOP normal distribution, the mean of TOP distribution is thus the pressure at which maximum rate of recruitment occurs during inflation and that mean of the total lung units can be recruited if ventilated at that pressure. Similarly, in the TCP distribution, the mean TCP indicates the maximum rate of derecruitment during passive deflation and also mean lung units that will remain recruited during deflation. The standard deviation (SD) of the distribution, in combination with the mean TOP and TCP is therefore, an estimate of the heterogeneity of the lung. SD values will change for a given animal depending on "how injured" the lung is.

Examples of how different lung conditions affecting the lung unit TOP distribution are shown in Fig 3(a) and 3(b). The upper Fig section is an example of the PV curve and the bottom section shows the corresponding TOP distribution. A collapsed lung requires an overall higher pressure to open/ 
recruit the lung units, therefore, mean TOP will increase as shown in Fig 3(a). The SD value is the "spread" of the TOP and TCP distribution and thus, a more heterogeneous lung will result in a higher SD value, and with wider spread of the effective TOP distribution in Fig 3(b). Similar concept applies to TCP distribution.

a)
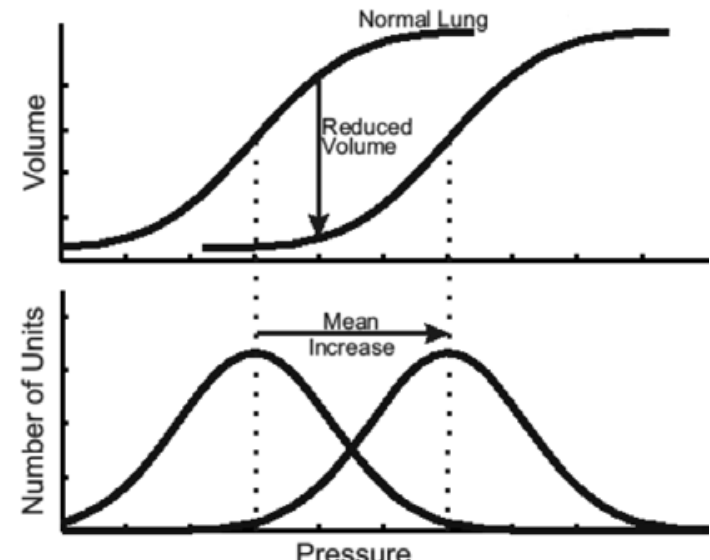

b)
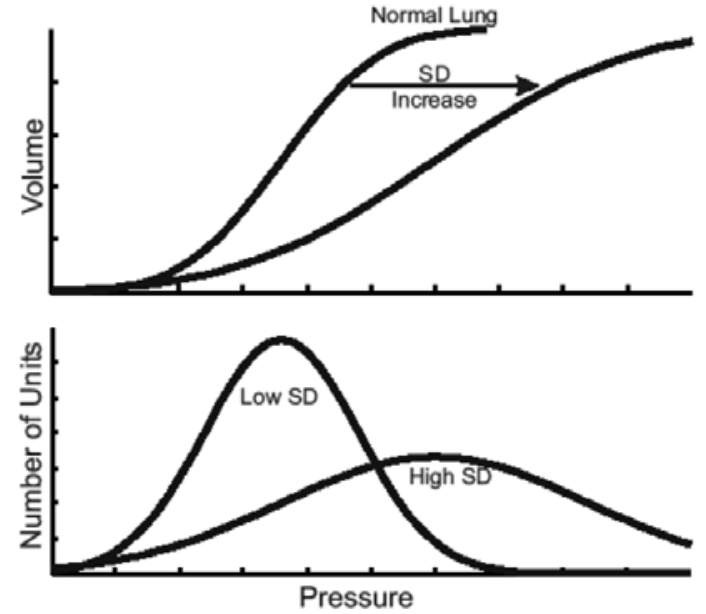

Fig. 3. Effect of TOP and SD towards a PV curve (Top - PV curve during inflation, Bottom - TOP distribution based on PV curves). (a) Normal lung to collapse Lung. (b) Normal lung to heterogeneous lung.

In this study, the measured PV curves were fitted with the minimal model and the fitting errors during inflation were calculated and presented as mean absolute percentage error to check the model performance in both healthy and ARDS animal. Model-based estimated mean TOP, TCP and SD in both healthy and ARDS states were individually compared to examine the effect of ARDS towards the parameters and their physiological relevance.

\section{RESULTS}

9 pigs weighing median 24.0kg [IQR: 21.0-29.6] were included in the study. 3 animals reached an ARDS state after oleic acid injection and comparisons were made between healthy (HS) and ARDS state (AS).

Table 1 shows the model absolute percentage fitting error during inflation for all healthy animals. Table 2 shows the fitting error for the 3 animals which are initially healthy and later developed ARDS.
Table 3 shows the model estimated mean TOP at different PEEP values, along with the SD of the TOP distribution during healthy and ARDS state. Table 4 shows the model estimated mean TCP and SD for the 3 animals during healthy and ARDS state at each PEEP.

Table 1. Absolute percentage fitting error during inflation in each PEEP for animals in healthy state (HS)

\begin{tabular}{|c|c|c|c|c|c|}
\hline & \multicolumn{4}{|c|}{ Model Fitting Error (\%) at PEEP } & \multirow{2}{*}{ Median [IQR] } \\
\cline { 2 - 5 } & $\mathbf{5}$ & $\mathbf{1 0}$ & $\mathbf{1 5}$ & $\mathbf{2 0}$ & \\
\hline HS1 & 32.48 & 2.19 & 1.56 & 0.72 & $1.88[1.35-9.76]$ \\
\hline HS2 & 24.94 & 2.08 & 2.25 & 1.71 & $2.17[1.98-7.93]$ \\
\hline HS3 & 24.62 & 7.43 & 3.05 & 2.63 & $5.24[2.95-11.73]$ \\
\hline HS4 & 6.46 & 5.97 & 2.66 & 0.84 & $4.31[2.20-6.09]$ \\
\hline HS5 & 12.45 & 9.56 & 2.25 & 0.31 & $5.91[1.77-10.28]$ \\
\hline HS6 & 27.46 & 4.81 & 3.11 & 0.86 & $3.96[2.55-10.47]$ \\
\hline HS7 & 9.96 & 3.36 & 2.49 & 0.53 & $2.92[2.00-5.01]$ \\
\hline HS8 & 15.45 & 3.36 & 2.74 & 1.09 & $3.05[2.33-6.38]$ \\
\hline HS9 & 10.93 & 5.25 & 2.75 & 0.36 & $4.00[2.15-6.67]$ \\
\hline Median & 15.45 & 4.81 & 2.66 & 0.84 & \multirow{2}{*}{$2.90[1.98-7.97]$} \\
[IQR] & {$[10.93-$} & {$[3.36-$} & {$[2.25-$} & {$[0.53-$} & \\
& $24.94]$ & $5.97]$ & $2.75]$ & $1.09]$ & \\
\hline
\end{tabular}

Table 2. Absolute percentage fitting error in each PEEP for animal in ARDS state (AS)

\begin{tabular}{|c|c|c|c|c|c|}
\hline & \multicolumn{4}{|c|}{ Model Fitting Error (\%) at PEEP } & \multirow{2}{*}{ Median [IQR] } \\
\cline { 2 - 5 } & $\mathbf{5}$ & $\mathbf{1 0}$ & $\mathbf{1 5}$ & $\mathbf{2 0}$ & \\
\hline AS1 & 5.81 & 7.27 & 3.08 & 1.08 & $4.45[2.58-6.18]$ \\
\hline AS2 & 2.85 & 5.64 & 4.13 & 0.69 & $3.49[2.31-4.51]$ \\
\hline AS3 & 41.44 & 9.62 & 2.17 & 3.63 & $6.62[3.27-17.57]$ \\
\hline
\end{tabular}

Table 3. Model mean TOP and SD inflation at each PEEP

\begin{tabular}{|l|c|c|c|c|c|}
\hline & \multicolumn{4}{|c|}{ TOP at PEEP $\left(\mathbf{c m H}_{\mathbf{2}} \mathbf{O}\right)$} & \multirow{2}{*}{ SD } \\
\cline { 2 - 5 } & $\mathbf{5}$ & $\mathbf{1 0}$ & $\mathbf{1 5}$ & $\mathbf{2 0}$ & \\
\hline HS1 & 42.6 & 32.7 & 24.8 & 19.2 & 23 \\
\hline HS2 & 31.3 & 28.1 & 23.8 & 21.5 & 14 \\
\hline HS3 & 38.2 & 33.9 & 29.4 & 22.8 & 21 \\
\hline AS1 & 48.1 & 44.1 & 33.3 & 22.7 & 25 \\
\hline AS2 & 49.5 & 41.6 & 31.1 & 19.1 & 25 \\
\hline AS3 & 68.1 & 64.7 & 58.7 & 49.4 & 23 \\
\hline
\end{tabular}

Table 4. Model mean TCP and SD deflation at each PEEP

\begin{tabular}{|l|c|c|c|c|c|}
\hline & \multicolumn{4}{|c|}{ TCP at PEEP $\left(\mathbf{c m H}_{\mathbf{2}} \mathbf{O}\right)$} & \multirow{2}{*}{ SD } \\
\cline { 2 - 5 } & $\mathbf{5}$ & $\mathbf{1 0}$ & $\mathbf{1 5}$ & $\mathbf{2 0}$ & \\
\hline HS1 & 10.2 & 13.2 & 15.7 & 18.4 & 4 \\
\hline HS2 & 9.0 & 12.5 & 15.5 & 18.8 & 3 \\
\hline HS3 & 8.7 & 12.3 & 15.8 & 19.3 & 3 \\
\hline AS1 & 10.2 & 14.0 & 16.7 & 19.0 & 4 \\
\hline AS2 & 10.2 & 13.7 & 16.7 & 18.9 & 4 \\
\hline AS3 & 9.6 & 14.1 & 18.2 & 21.8 & 3 \\
\hline
\end{tabular}

Fig 4 shows the model fitting results in a animal (HS2) estimating the TOP and TCP distribution at PEEP $=10$ and $15 \mathrm{cmH}_{2} \mathrm{O}$. An example of PV shift from the healthy to the ARDS state is shown in Fig 5 (HS1 to AS1). The comparison 
of mean TOP between healthy and ARDS for 3 animals is shown in Fig 6.

a)

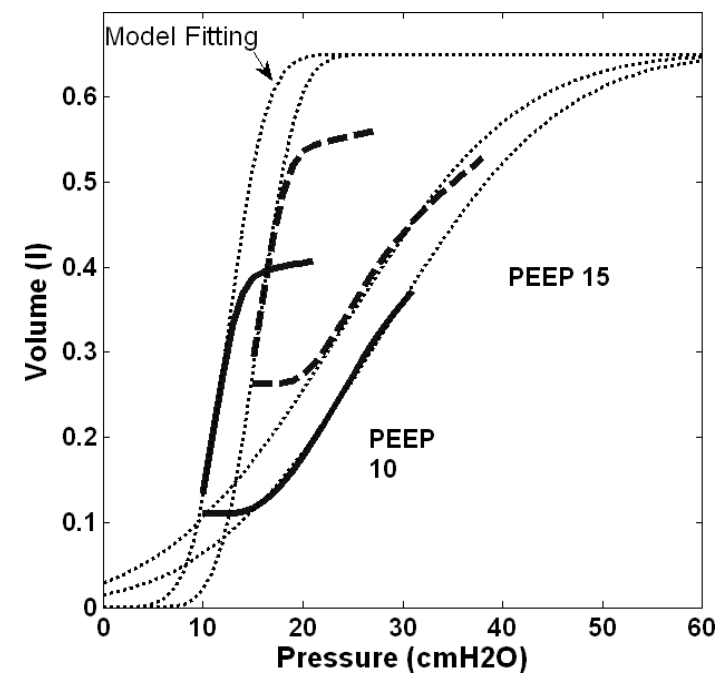

b)

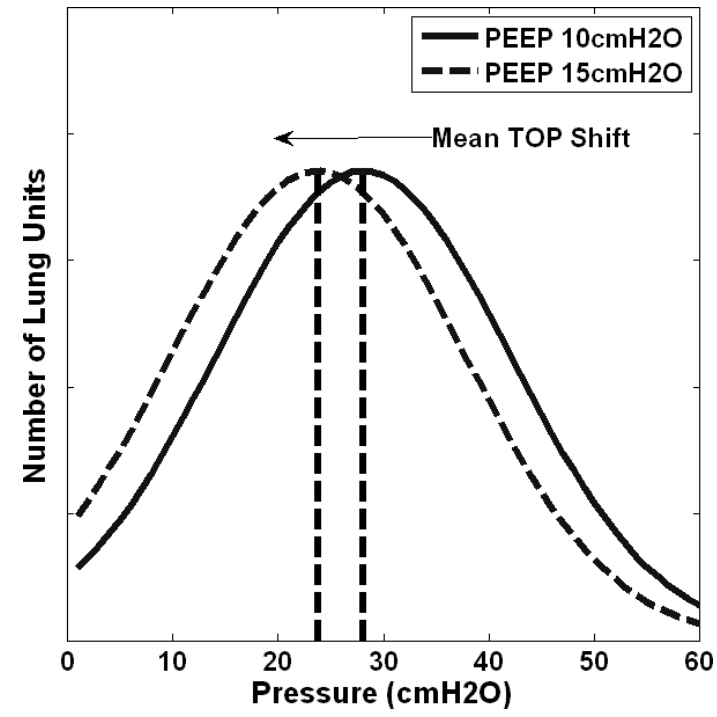

c)

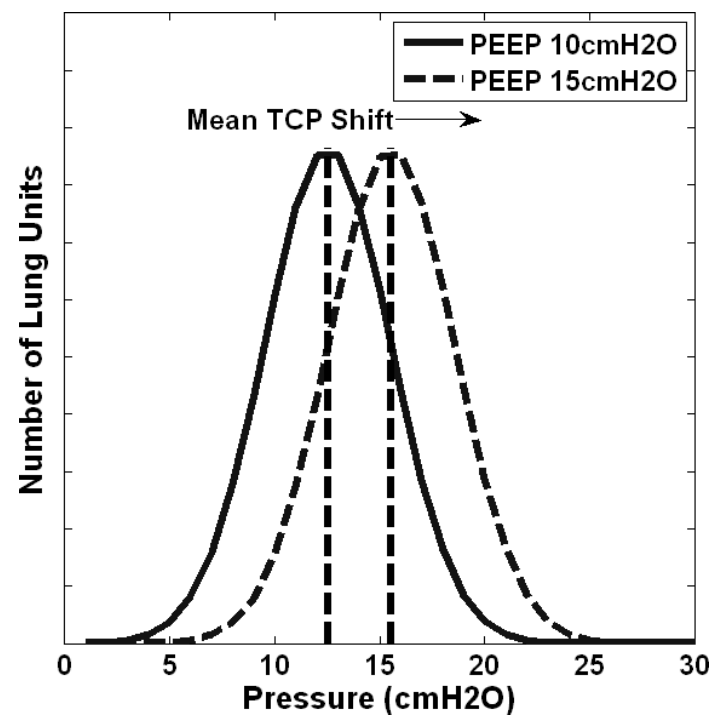

Fig. 4. (a) Model fitting (b) mean TOP shift and (c) mean TCP shift with PEEP increase. (Thick lines in (a) indicate post-process measured PV curves)

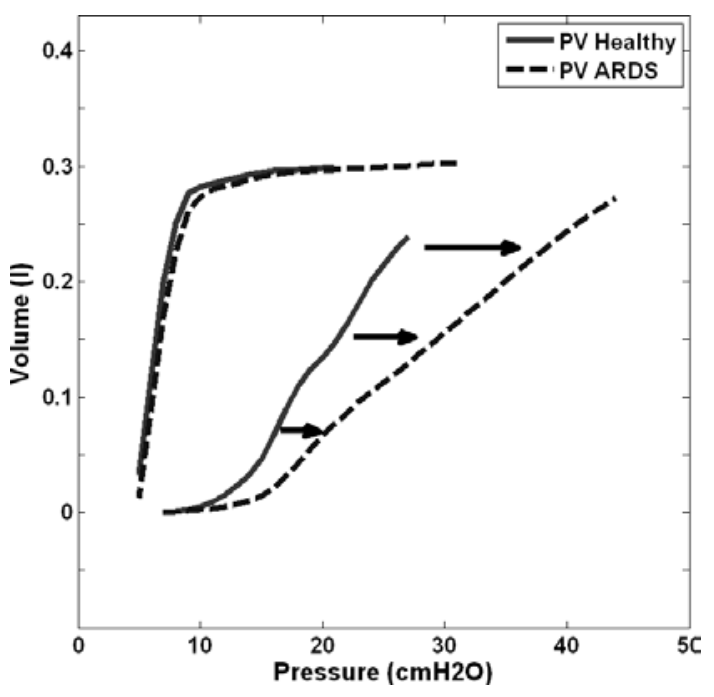

Fig. 5. PV curve shift from Healthy to ARDS.

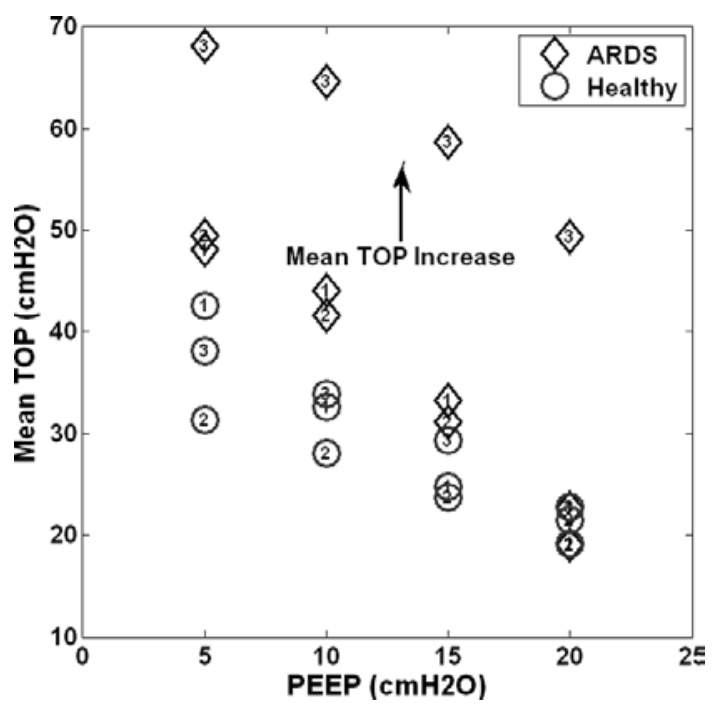

Fig. 6. Comparison of estimated mean TOP in healthy and ARDS state.

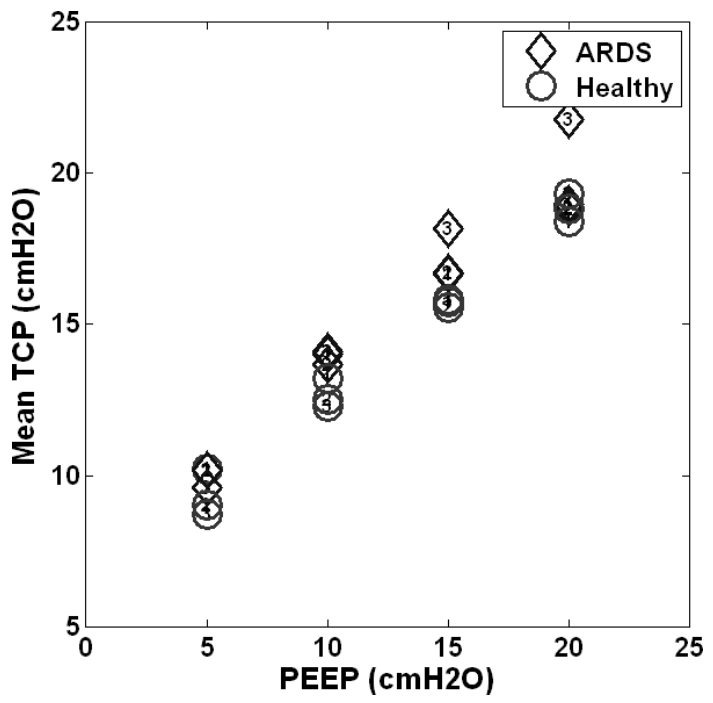

Fig. 7. Comparison of estimated mean TCP in healthy and ARDS state. 


\section{DISCUSSIONS}

The overall median [IQR] fitting error for the minimal model in 9 healthy animals is $2.90 \%$ [IQR: $1.98-7.97]$. An example of model fitting to the measured PV curve is shown in Fig 4(a). Similar to healthy animals, the model fits well for ARDS in the same animal with median absolute percentage error less than $7 \%$.

The minimal model fits better when animals are ventilated at higher PEEP $(\mathrm{P}<0.005)$ compared to lower PEEP, as shown in Table 1 and Table 2. There is a noticeable high fitting error for each animal at PEEP $5 \mathrm{CmH}_{2} \mathrm{O}$, and up to $41.44 \%$ (AS3 at Table 2) during inflation. It is hypothesized that the model was not able to accurately capture the physiological conditions at low PEEP. In particular, this error can be associated with the effect of intrinsic or auto PEEP. AutoPEEP could be readily added for this model to be factored and accounted for. However, the relatively low median error indicates that the model was capable of capturing fundamental mechanics of both healthy and ARDS lungs.

Tables 3 shows the estimated mean TOP for all 3 animals during the healthy and ARDS state. In the healthy state, the overall TOP at PEEP $=5 \mathrm{cmH}_{2} \mathrm{O}$ is higher at $42.4 \mathrm{cmH}_{2} \mathrm{O}$ [IQR: 38.2-44.9] than 25.0 $\mathrm{cmH}_{2} \mathrm{O}$ [IQR: 21.5-27.1] at higher PEEP at $20 \mathrm{CmH}_{2} \mathrm{O}$. The TOP shift of an animal during PEEP change can be observed in Fig 4(b). The lowering of mean TOP with PEEP increase indicates that more alveoli are recruited. Similar TOP trends are also observed in ARDS animals, but an overall higher TOP is observed compared to healthy animals, which is also expected for a stiffer ARDS lung. Fig 4(c) shows mean TCP increases with PEEP as expected suggesting that higher PEEP will retain recruited lung units and prevent them from collapsing.

Healthy lungs normally consist of only opened or recruited lung units, and it might be assumed that the concept of recruitment is not applicable. However, during inflation of a healthy lung, pressure is still needed to ventilate the already opened lung units and the pressure is relatively lower compared to the pressure required to fill and recruit a collapsed ARDS affected lung unit. Therefore, the area within the PV curve for a healthy lung is 'smaller' than ARDS lung. Fig 5 shows a clear comparison of a healthy and ARDS PV curve, in which the ARDS PV curve is 'bigger' than healthy PV curve. The change thus shows the expected higher work of breathing in ARDS.

Comparing the mean TOP of healthy and ARDS, it is found that estimated TOP for healthy lungs are lower when compared to ARDS lungs in Fig 6. A healthy lung is a less heterogeneous lung and the effect of superimposed pressure to alveoli is less detrimental. As suggested earlier, a healthy lung is normally open, which results in a reduction of mean TOP. Thus, the model captures the fact that, for the same animal at a healthy and ARDS state, higher pressure is required to recruit and open the lung. Hence, the clinical use of PEEP to monitor recruitment and treat ARDS patients. Overall, this model matches clinical observation and expectation, further validating the model.
For the ARDS animals, it was also observed that the SD increased after the animals were diagnosed with ARDS (Table 3). This increase is expected and indicates the change in lung heterogeneity and loss of compliance. The loss of compliance thus requires a higher work of breathing to ventilate the animal resulting in the bigger PV curve.

Theoretically, mean TCP should be higher in ARDS state compared to healthy state. ARDS lung units are more unstable and vulnerable to collapse, thus higher pressure is required to retain the recruitment. However, this condition was neither observed nor apparent in the study results. It is found that the deflation curve for ARDS is similar to healthy animals as shown in Fig 5, which results in relatively no change in TCP and SD. The results in Table 4 and Fig 7 show the same trend and, it is suggested that deflation of the lung is dependant on the patient's unloading, and the lung stiffness or compliance will have little or no effect during this cycle. Deflation occurs during the patients respiratory unloading and is dependent on airway resistance. If the airway resistance remains constant throughout the trial, the deflation cycle will be the same regardless of the animal state, and equally, mean TCP and SD will not change for the animal.

A decrease in mean TOP with PEEP increase will indicate additional alveoli being recruited. Thus, the trend where mean TOP decrease with increasing PEEP is less significant, shows that additional PEEP recruit relatively little alveoli with additional pressure. Thus, mechanical ventilation PEEP can be selected at point where maximum recruitment (Significant mean TOP drop) occurs (Sundaresan et al., 2011).

\section{LIMITATIONS}

After oleic acid injections, only 3 animals of 9 successfully developed ARDS. The other animals experienced hemodynamic failure before ARDS could develop fully. This result shows that oleic acid induced ARDS animals are less reproducible and the animal preparation method should be reexamined (Julien et al., 1986, Grotjohan et al., 1996, Schuster, 1994, Rosenthal et al., 1998). The comparison for estimated TOP, TCP and SD between healthy and ARDS is thus, not conclusive with statistical significance given low animal numbers. However, individual data revealed that animals that developed ARDS had overall higher TOP compared to animal in a healthy state. This physiologically relevant result is supported by past literatures which examine similar clinical conditions (Lu et al., 2001, Crotti et al., 2001, Pelosi et al., 2001). In addition, all other results follows clinically expected trends. Future ongoing trials can be carried out to test the significance of the findings.

Another limitation is that the oleic acid ARDS animal model in this study only captures specific ARDS condition. More specifically, the ARDS model does not fully represent the actual ARDS patients, and the estimated TOP values in this study can be overestimated or vice-versa, compared to actual clinical settings. The trends of TOP mean shift with PEEP thus, carries more valuable information as it is a representation of recruitment. 
Overall, the difference of mean TOP and SD between healthy and ARDS state can be identified using the minimal model. This allows a comparison between both healthy and ARDS states and potential application of minimal model in the ICU to monitor patients-specific condition to guide MV therapy. Mean TCP however, have little change between healthy and ARDS state indicating that the TCP parameter was less significant in disease state tracking.

\section{CONCLUSIONS}

The minimal model fits well in healthy and ARDS lungs, and is capable of capturing the fundamental lung mechanics of the healthy and ARDS lung. The application of minimal model is thus not limited to diseased lung cases, and can be used to monitor healthy lungs in sedated patients. The model estimated parameters allows disease state tracking, which in turn reveals a potential to assist in clinical decision making.

\section{REFERENCES}

Chase, J. G., Le Compte, A., Preiser, J.-C., Shaw, G., Penning, S. and Desaive, T. (2011). Physiological modeling, tight glycemic control, and the ICU clinician: what are models and how can they affect practice? Annals of Intensive Care, volume 1:11.

Crotti, S., Mascheroni, D., Caironi, P., Pelosi, P., Ronzoni, G., Mondino, M., Marini, J. J. and Gattinoni, L. (2001). Recruitment and Derecruitment during Acute Respiratory Failure . A Clinical Study. Am J Respir Crit Care Med, volume 164, 131-140.

Grotjohan, H. P., Van Der Heijde, R. M. J. L., Jansen, J. R. C., Wagenvoort, C. A. and Versprille, A. (1996). A stable model of respiratory distress by small injections of oleic acid in pigs. Intensive Care Medicine, volume 22, 336-344.

Hickling, Keith g. (1998). The Pressure-Volume Curve Is Greatly Modified by Recruitment . A Mathematical Model of ARDS Lungs. Am. J. Respir. Crit. Care Med., volume 158, 194-202.

Julien, M., Hoeffel, J. M. and Flick, M. R. (1986). Oleic acid lung injury in sheep. Journal of Applied Physiology, volume 60 , 433-440.

Lu, Q., Malbouisson, L. M., Mourgeon, E., Goldstein, I., Coriat, P. and Rouby, J. J. (2001). Assessment of PEEPinduced reopening of collapsed lung regions in acute lung injury: are one or three CT sections representative of the entire lung? Intensive Care Medicine, volume 27, 1504-1510.

Pelosi, P., Goldner, M., Mckibben, A., Adams, A., Eccher, G., Caironi, P., Losappio, S., Gattinoni, L. and Marini, J. J. (2001). Recruitment and derecruitment during acute respiratory failure: an experimental study. Am J Respir Crit Care Med, volume 164, 122 - 30.

Rees, S., Allerød, C., Murley, D., Zhao, Y., Smith, B., Kjærgaard, S., Thorgaard, P. and Andreassen, S. (2006). Using physiological models and decision theory for selecting appropriate ventilator settings. Journal of Clinical Monitoring and Computing, volume 20, 421429.
Rosenthal, C., Caronia, C., Quinn, C., Lugo, N. and Sagy, M. (1998). A comparison among animal models of acute lung injury. Critical Care Medicine, volume 26, 912916.

Schranz, C., Knobel, C., Kretschmer, J., Zhao, Z. and Moller, K. (2011). Hierarchical Parameter Identification in Models of Respiratory Mechanics. IEEE Trans Biomed Eng, volume.

Schuster, D. (1994). ARDS: clinical lessons from the oleic acid model of acute lung injury. Am. J. Respir. Crit. Care Med., volume 149, 245-260.

Sundaresan, A., Chase, J., Shaw, G., Chiew, Y. S. and Desaive, T. (2011). Model-based optimal PEEP in mechanically ventilated ARDS patients in the Intensive Care Unit. BioMedical Engineering OnLine, volume 10, 64.

Sundaresan, A., Yuta, T., Hann, C. E., Geoffrey Chase, J. and Shaw, G. M. (2009). A minimal model of lung mechanics and model-based markers for optimizing ventilator treatment in ARDS patients. Computer Methods and Programs in Biomedicine, volume 95, 166180. 